\title{
High-speed Measurement of Vibrational and Rotational Temperatures of Nitrogen Molecules behind Hypervelocity Shock Wave by CARS Method
}

\author{
By Kazuo MAENO'), ${ }^{1)}$ ouichi ENDO ${ }^{2)}$, Keishi ARIMURA ${ }^{2)}$, Takashi OSADA ${ }^{2)}$, and Masanori OTA ${ }^{3)}$ \\ 1) Graduate Course \& Department of Mechanical Engineering, Graduate School of Engineering \\ ${ }^{2)}$ Graduate Student, Graduate School of Engineering \\ ${ }^{3)}$ Graduate Course \& Department of Urban Environment Systems, Graduate School of Engineering \\ Chiba University, Chiba, Japan \\ (Received May 2nd, 2008)
}

In the re-entering phase of space vehicle into the atmosphere strong shock wave is generated in front of the hypersonic vehicle, and high enthalpy flow with radiating, non-equilibrium real gas effects is generated behind the shock wave. As well as usual convecting heat transfer, the radiative and non-equilibrium real gas heating from the shock-heated air ahead of the vehicle plays an important role on the total heat flux to the wall surface, which affects the heat shield design of the re-entering vehicle. As for the research of such non-equilibrium high enthalpy flows behind strong shock waves, several spectroscopic studies have been reported by using shock tubes or shock tunnels. The direct measurement, however, of molecular vibrational/rotational temperatures from ground state, has not been performed, and the experimental data are in quite insufficient conditions. In this paper nonlinear spectroscopic measurement is performed on the vibrational/rotational temperatures of nitrogen behind the hypervelocity shock waves from $4-5 \mathrm{~km} / \mathrm{s}$ by CARS method. The CARS spectroscopic signals have been successfully observed by free-piston, double-diaphragm shock tube experiments, and the measured results are compared with the simulated spectra to decide the temperatures by spectral fitting method.

Key Words: CARS, Hypervelocity Shock Wave, Vibrational and Rotational Temperatures, Nitrogen Molecule, Spectral Fitting

\section{Introduction}

The radiative high enthalpy flow is of great importance in the research of heat shield problems for re-entering space vehicles into the atmosphere. The non-equilibrium flow with radiative and real gas effects from the shocked air ahead of the vehicle plays an important role on the total heat flux to the wall surface as well as usual convective heat transfer. In order to clarify the radiative and non-equilibrium characteristics of the strongly shocked air, many experimental studies have been carried out during the last decades by the researchers using shock tube devices ${ }^{1), 2)}$.

In our previous works a CCD camera system was introduced in the radiation observation of hypervelocity shock waves in low-density air ${ }^{3)}$ with an imaging spectrograph, a streak camera, or a gated image-intensified CCD (ICCD) camera and personal computer. Some interesting features were obtained independently for the total radiation and for the spectral radiation behind shock waves with velocity over 10 $\mathrm{km} / \mathrm{s}$ in air $^{4)}$. Based on these experimental results, we also used the Boltzmann plot method related to the second positive band of nitrogen molecules to evaluate vibrational/rotational temperatures of the molecule from the time-resolved spectroscopic data. The Boltzmann plot method, however, was based on several assumptions, and the method suffered from experimental peak to peak errors and measured data of the activation from different state levels apart from ground state of observed molecules.

On the other hand, as for the combustion experiments, Coherent Anti-Stokes Raman Spectroscopy (CARS) method has been developed for temperature and concentration measurement method $^{5,6)}$ in connection to the number density of observed species. The CARS has been successfully used with a nonlinear, four-wave optical mixing technique for high temperature flows, combustion, and so on ${ }^{7), 8), 9)}$. The CARS system also produces a strong and coherent signal that can be detected even in the strong background luminosity.

In our study CARS measurement of vibrational/rotational temperatures of nitrogen molecules in hypersonic air flow behind the strong shock waves has been established over 5 $\mathrm{km} / \mathrm{s}$ shock velocity ${ }^{10)}$. The CARS signal could be successfully acquired from the non-equilibrium hypervelocity flow behind the strong shock waves with 10ns gate range. In this paper the measured CARS results for shock waves from $4 \mathrm{~km} / \mathrm{s}$ to above $5 \mathrm{~km} / \mathrm{s}$ are reported. In addition, these spectroscopic data are compared with the theoretical spectroscopic data, and vibrational/rotational temperatures are evaluated for different shock wave velocity by spectral fitting method. We also have evaluated the spectral matching errors to improve the reliability of our CARS experiments.

\section{CARS Method and Calculation}

CARS is one of the Raman spectroscopy methods. This method is spectroscopic diagnostics which can determine the vibrational/rotational temperatures and chemical species concentration. This spectroscopy method corresponds to the scattering phenomena for nonlinear response of the medium to electromagnetic wave. In CARS method two lasers are irradiated to a sample, then the strong emission intensity by 
scattering process is generated. The CARS has such features that the scattered light intensity is very high of $10^{5} \sim 10^{10}$ times than Raman scattered light intensity, and the signal is emitted in one direction as a laser beam, so the CARS has high space-time resolution. It has, however, issues that the arrangement of optical system and temporal matching are very much complicated and difficult.

The theory of the CARS effect has been developed extensively. Incident laser beams at frequencies $\omega_{1}$ (pumping beam) and $\omega_{2}$ (Stokes beam) interact through the third order nonlinear electric susceptibility $\chi^{(3)}$ CARs of the gas molecule to produce coherent radiation $\left(\omega_{3}\right)$ at $\omega_{3}=2 \omega_{1}-\omega_{2}$ as follows;

$$
I_{3}\left(\omega_{3}\right)=\frac{\omega_{3}^{2}}{n_{1}^{2} n_{2} n_{3} c^{4} \varepsilon_{0}^{2}}\left|\chi_{C A R S}^{(3)}\right|^{2} I_{1}^{2}\left(\omega_{1}\right) I_{2}\left(\omega_{2}\right) l^{2}\left(\frac{\sin \frac{\Delta k l}{2}}{\frac{\Delta k l}{2}}\right)^{2}
$$

$$
\Delta k=2 k_{1}-k_{2}-k_{3}
$$

where $n_{1}, n_{2}$ and $n_{3}$ are the refractive index at $\omega_{1}, \omega_{2}, \omega_{3}$, respectively; $c$ is the velocity of light; $l$ is interaction length; $k_{1}$, $k_{2}$ and $k_{3}$ are the wave vectors of the pumping, Stokes, and CARS beams, respectively; $\varepsilon_{0}$ is the permittivity of free space. The CARS signal is enhanced on the condition of phase matching, $\Delta k=0$. Then the four vectors form a phase-matching diagram (BOXCARS) shown in Fig.1. The incident laser beams, i.e. two pump beams $\left(k_{1}\right)$ and a Stokes beam $\left(k_{2}\right)$, are aligned in order to satisfy this vector relation. In this state the CARS signal can be obtained at high signal intensity. Moreover, emission intensity is proportional to the cube of incident beam (the square of pump beam and the first power of Stokes beam). $\chi^{(3)}$ CARS is shown by

$$
\chi_{C A R S}^{(3)}=\sum_{j} K_{j} \frac{\Gamma_{j}}{2 \Delta \omega_{j}-i \Gamma_{j}}+\chi_{n r}
$$

where the $j$ summation is over vibration-rotation transitions in the vicinity of $\omega_{1}-\omega_{2} ; \Gamma_{j}$ is the Raman line width (FWHM); $\chi_{n r}$ is a background contribution due to electrons and remote resonances; and $\Delta \omega_{j}$ is shown to be related to the Raman cross-section by ${ }^{9)}$

$$
K_{j}=\left.\frac{2 n_{1} c^{4}}{n_{2} \hbar \omega_{2}^{4}} N \Delta_{j} \frac{d \sigma}{d \Omega}\right|_{j} \Gamma_{j}^{-1}
$$

where $\hbar$ is $h$ devided by $2 \pi, N$ is the number density of the Raman active molecule, $\Delta_{\mathrm{j}}$ is the population difference between the upper and lower vibration-rotation states, and $\left.(d \sigma / d \Omega)\right|_{j}$ is the cross-section for spontaneous Raman scattering.

On the assumption that molecules have Boltzmann distributions based on the rotational $\left(T_{r}\right)$ and vibrational $\left(T_{v}\right)$ temperatures, $\Delta_{j}$ can be expressed as

$$
\begin{aligned}
\Delta_{j}= & \frac{(2 J+1) g_{I}}{Q_{r} Q_{v}}\left[\exp \left(\frac{-F_{v, J} h c}{k T_{r}}\right) \exp \left(\frac{-G_{v} h c}{k T_{v}}\right)\right. \\
& \left.-\exp \left(\frac{-F_{v+1, J} h c}{k T_{r}}\right) \exp \left(\frac{-G_{v+1} h c}{k T_{v}}\right)\right]
\end{aligned}
$$

where $F_{v, j}$ and $G_{v}$ are the rotational and vibrational energy terms, respectively; $g_{l}$ is the spin degeneracy, 6 for even- $J$ rotational levels and 3 for odd- $J$ levels in $\mathrm{N}_{2}$; and $Q_{r}$ and $Q_{v}$ are the rotational and vibrational partition functions, respectively.

From Eqs. (1), (3) and (4), CARS signal may be approximately proportional to the square of number density of molecule. Therefore, it is not easy to detect the CARS signal especially from low-pressure and radiating gas of fast velocity. Figure 1 is the vector diagram of BOXCARS. When this vector relation is attained in each pumping laser beams $\mathbf{k}_{1}$ and Stokes beam $\mathbf{k}_{2}$, the measured CARS signal $\mathbf{k}_{3}$ becomes coherent and strong.

As written in the following section, the measured CARS spectra are fitted with calculated CARS spectra by treating $T_{v}$, $T_{n}, \Gamma$ as free parameters to decide the temperatures.

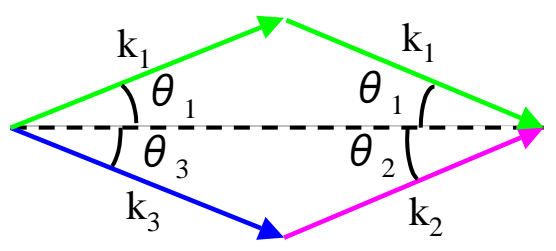

Fig. 1. Phase-vector diagram of BOXCARS

\section{Spectral Fitting Method for Temperature Estimation}

CARS spectroscopic data obtained by our experiment relate to the energy levels which correspond to the kinetic conditions of the molecule. Vibrational/rotational temperatures are determined by fitting the computer-generated and theoretical spectra to the observed CARS spectra. The spectral fitting method is explained as follows. First, theoretical spectra are developed by the computer program, which includes the vibrational/rotational temperatures, the Raman line widths and non-resonant parameters, and these parameters are varied in the assigned bounds. Secondly, square mean errors of normalized emission intensity between the computergenerated theoretical spectrum and observed CARS spectrum are calculated, and finally vibrational/rotational temperatures of nitrogen molecule are determined, where the error become minimum.

Raman line width corresponds to the half width at half maximum of Raman line, and the function of pressure, temperature and rotational quantum number of the molecule. Non-resonant parameter is the constant, unconcerned element about Raman resonance.

\section{Experimental Apparatus and Diagnostic System}

In CARS experiment we have used a free-piston, doublediaphragm shock tube to generate the strong shock waves in low-density gas as shown in Fig. $2^{11)}$. This shock tube consists of high-pressure tube (driver gas is nitrogen), compression tube (helium gas is supplied), buffer tube (supplied gas is also helium), low-pressure tube (test gas is air or nitrogen), and vacuum chamber. The each tube is divided off by a quick action valve, the first diaphragm (steel), and the second 
diaphragm (aluminum). The test section of the low-pressure tube, where the CARS laser beams are crossed, is $40 \mathrm{~mm} \times$ $40 \mathrm{~mm}$ square. The observation window of the test section is mounted near a focal lens with some distance from the sidewall of the two small halls along the optical path of laser beam. The shock velocity is measured using ion probes mounted on the inside wall of test-section. The experimental apparatus enables us to produce the strong shock waves by the usage of two diaphragms. The adiabatic compression by free piston of helium ruptures the steel diaphragm, and generates shock wave in buffer tube. Then by reflection of the shock wave and rupturing of aluminum diaphragm, the stronger shock wave is generated in the test section.

Figure 3 shows a layout of diagnostic system for the CARS measurement in shock waves (CARS system). CARS system consists of a doubled Nd: YAG laser $\left(\omega_{1}\right)$ of second harmonics, a dye laser $\left(\omega_{2}\right)$, a spectrograph, and an ICCD camera.

The Nd: YAG laser (Continuum PL8010) is used for the pumping beam $\left(\omega_{1}\right)$. At second harmonics beam $(532 \mathrm{~nm})$, the line width is $0.5 \mathrm{~cm}^{-1} \mathrm{FWHM}$, and the pulse duration is $10 \mathrm{~ns}$.

The laser beam $\left(\omega_{1}\right)$ is divided in two lines by a beam splitter (BS). These beams are combined to the dye laser beam $\left(\omega_{2}\right)$ by a beam combiner (BC2). Then these beams are focused at measurement point of observation section. The dye laser is adjusted wavelength due to pigment of compounding. Rhodamine 640 and Rhodamine 610 are dissolved by methanol (wave-length band: $\lambda=594 \mathrm{~nm} \sim 629 \mathrm{~nm}$ ). The dye laser, Stokes beam, is possible to oscillate at narrowband and broadband, in this study Stokes beam is selected to broadband. Stokes beam delays a few ns as excited by the YAG laser. So the optical path length of pumping beam is adjusted to synchronize arrival time of these beams at measurement point.

The CARS spectrum is detected by the spectrograph and the ICCD camera. The entrance slit width is set to $100 \mu \mathrm{m}$ throughout the observation. The ICCD camera is mounted on the focal exit of the spectrograph.

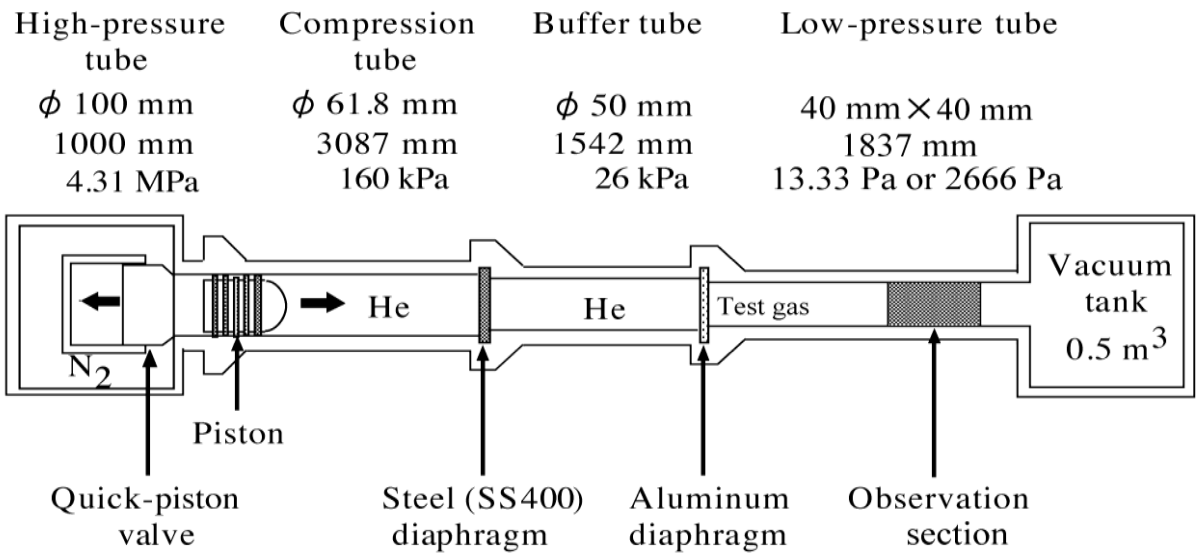

Fig. 2. Free-piston \& double-diaphragm shock tube

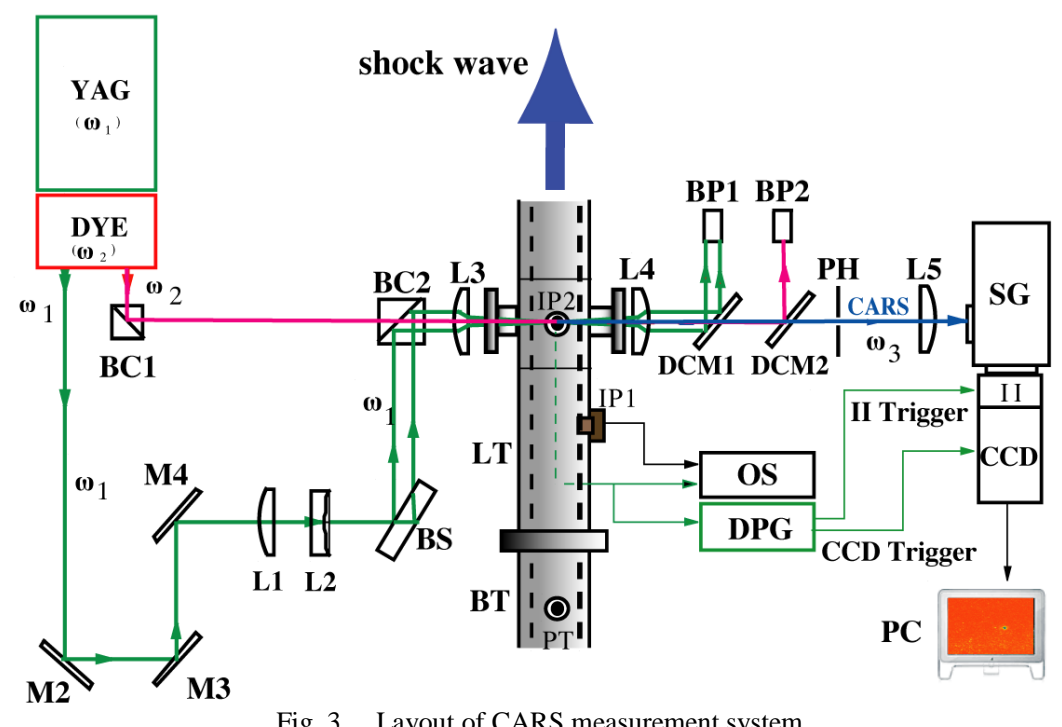

Fig. 3. Layout of CARS measurement system

Code: HN, He-Ne laser; M, mirror; BC, beam combiner; L, lens; BS, beam splitter; DCM, diachronic mirrors; BP, beam pocket; PH, pine hole; IP, ion probe; PT, pressure transducer; DPG, delay pulse generator; SG, spectrograph; OS, oscilloscope; LT, low-pressure tube; BT, buffer tube. 
Two pumping beams and Stokes beam are focused into shock tube through a focal lens. The path of laser beams and CARS beam are shown in Fig.4. In this case phase-matching condition has to be satisfied as well as planer BOXCARS. This method is called as folded BOXCARS, especially as multiplex folded BOXCARS using broadband Stokes beam at single shot. This method has the advantage in separating CARS signal from pumping and Stokes beams.

In this study the synchronization of the shock wave and laser beams is very difficult to apply CARS method from the hypervelocity flow behind the strong shock wave. In this study, as the shock wave velocity ranges over $4-5 \mathrm{~km} / \mathrm{s}$, measurement accuracy should be within on order of $\mu$ s.

The three beams $\left(\omega_{1}\right.$ and $\left.\omega_{2}\right)$ must be irradiated on time when the shock wave running over $5 \mathrm{~km} / \mathrm{s}$ just arrives at the observation window. The trigger system for synchronizing laser beams with shock wave is shown in Fig.5. In this figure the charge and fire (flash) trigger signals are constantly applied with designated interval as indicated in dashed lines, and then at the shock tube operation real trigger system is applied as shown in solid lines. The $\mathrm{Nd}$ : YAG laser is controlled by external signals. To begin with, a charge trigger is applied for flash lamp in YAG laser. After the fire trigger a Q-switch trigger opens Q-switch, and starts the laser beam irradiation. Before the measurement the charge and flash triggers are repeated at $10 \mathrm{~Hz}$. To produce the shock wave, the

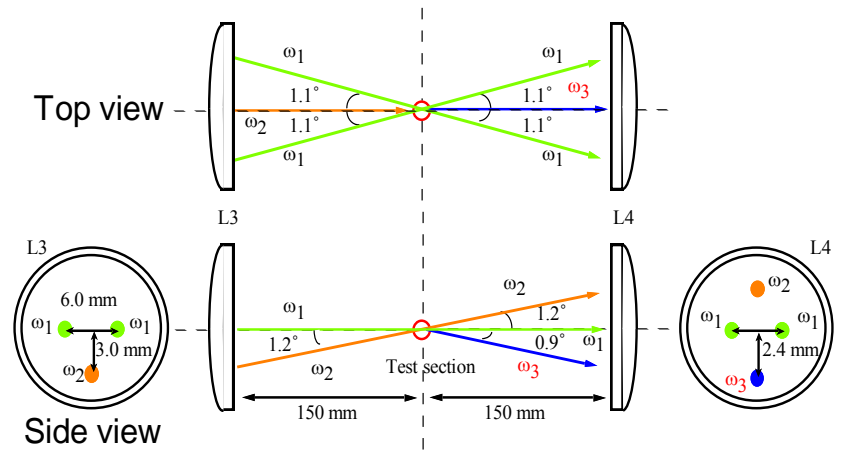

Fig. 4. Laser beams CARS path (top view and side view)

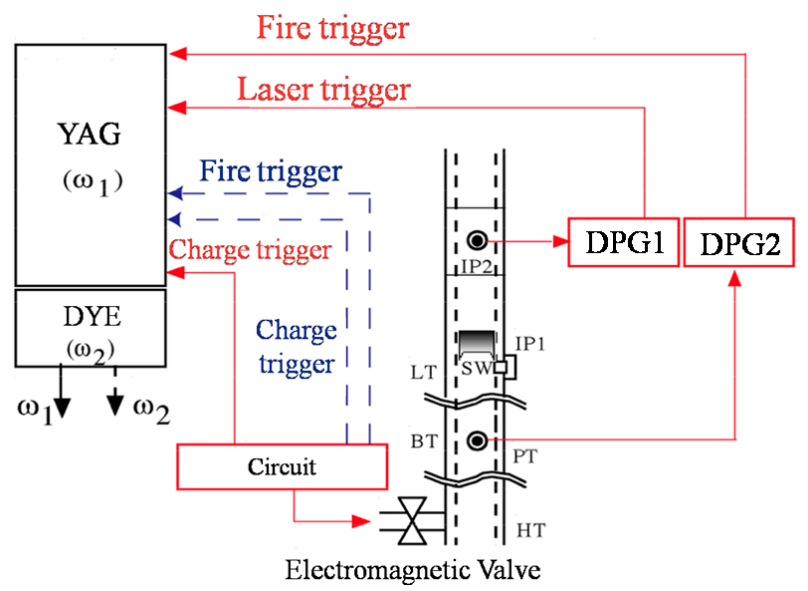

Fig. 5. Outline of the trigger system synchronizing laser beams with hypervelocity shock wave starting valve installed in a high-pressure tube is opened, and simultaneously the signal sends a charge trigger to the YAG laser. When shock wave arrives at a pressure transducer on the buffer tube, a delay pulse generator is triggered. Finally, DPG1 is triggered by short-circuit of an ion probe with shock wave passing, and sends a Q-switch trigger to YAG laser. As shown in Fig. 5, DPG1 sends simultaneously triggers to the ICCD for detecting CARS signal.

\section{Experimental Results and Discussion}

In this paper the measured spectral data are presented at $2666 \mathrm{~Pa}$ and $1333 \mathrm{~Pa}$ initial pressures. Reliability is evaluated for the measured spectra at $2666 \mathrm{~Pa}$ initial conditions. After comparing of spectral data by different shock wave velocities, the spectral fitting for each spectrum has been conducted.

The measured image of CARS signal is shown in Fig.6. Test gas is room air, and velocity of shock wave is $4.14 \mathrm{~km} / \mathrm{s}$ with Mach number of 12.04. The measurement position is $5.1 \mathrm{~mm}$ behind the shock front. Initial pressure is $2666 \mathrm{~Pa}$. Horizontal axis is wavelength and vertical axis is equivalent to the height of incidence light into the slit of spectrograph. The color is corresponding to the strength color bar on the right side. As shown in Fig.6, the clear image is taken without strong noise. Background light of YAG laser can be cut well.

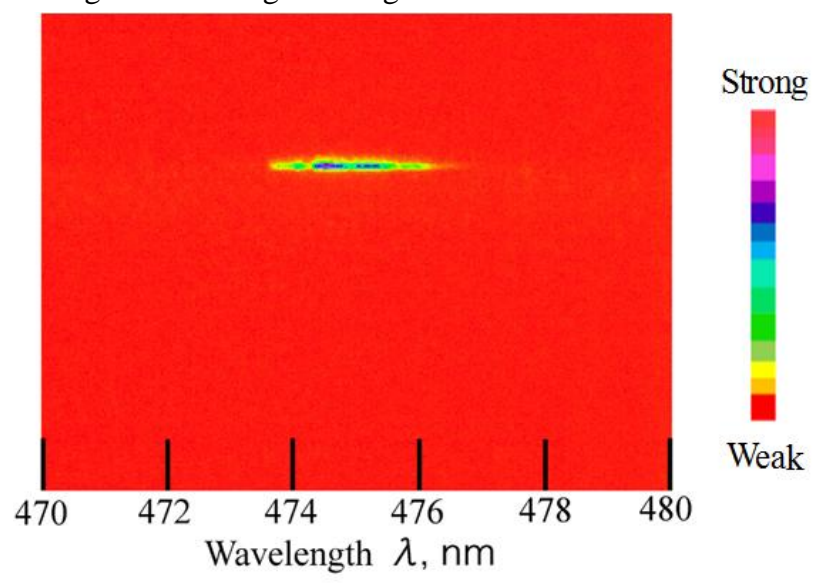

Fig. 6. Measured ICCD image of CARS signal

\subsection{Reliability of the spectrum}

Figure 7 shows the measured spectroscopic data. Horizontal axis is wave number and vertical axis is normalized emission intensity. The measured spectrum, Experiment1, corresponds to the image of CARS signal of Fig.6. Shock wave velocity of Experiment2 is $4.07 \mathrm{~km} / \mathrm{s}$, Mach number of 11.84 . The measurement position is $5.05 \mathrm{~mm}$ behind the shock front. Initial pressure is $2666 \mathrm{~Pa}$. As the experimental conditions are almost similar to both spectral data, reliability of the spectrum can be confirmed from these data. The spectral shape corresponds each other over $21150 \mathrm{~cm}^{-1}$ of spectrum range, without considering the non-resonant part. The peak and depth values and their wave numbers coincide well. In Experiment2 some noises are observed, so there are some differences of spectra to be influenced. Therefore, these spectra are swayed in some amount by background noises. Though the same 
experimental conditions are difficult for us to obtain, reliable data can be observed if clear spectral image is taken at same experimental conditions. So, the clear spectroscopic images without strong noises are needed to avoid mismatch in the spectral fitting procedure.

\subsection{Comparison of the spectral data by shock wave velocity}

The CARS spectrum of shock wave velocity of $4.14 \mathrm{~km} / \mathrm{s}$ (same as Experiment1 of Fig.7) is compared in Fig.8 with another spectrum from stronger shock wave velocity of $5.22 \mathrm{~km} / \mathrm{s}$, where the measurement position is $6.47 \mathrm{~mm}$ behind the shock front, Mach number is 15.1, and initial pressure is 1333Pa. The number of peaks is almost same, and the normalized height of peaks and depth of concaves agree partially, but the spectral data of shock wave velocity $5.22 \mathrm{~km} / \mathrm{s}$ shows totally larger intensity distribution. Especially, it is apparent that the spectrum of shock wave velocity $5.22 \mathrm{~km} / \mathrm{s}$ shows higher intensity at around 21030 and $21100 \mathrm{~cm}^{-1}$. It is not yet clarified which is the main cause between the difference of measuring positions behind the shock front, and difference of shock wave velocites, or simple error. Significant difference can be seen in non-resonant part

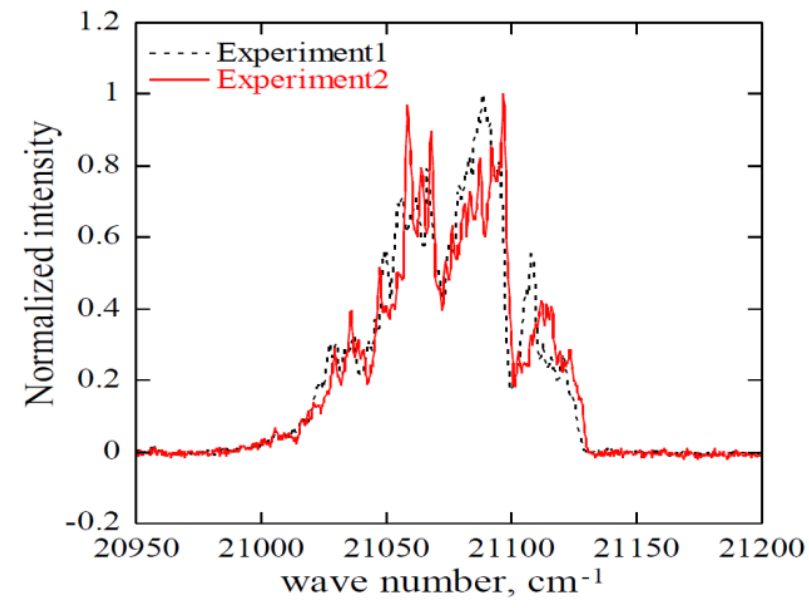

Fig. 7. Experimental spectra under the same experimental conditions

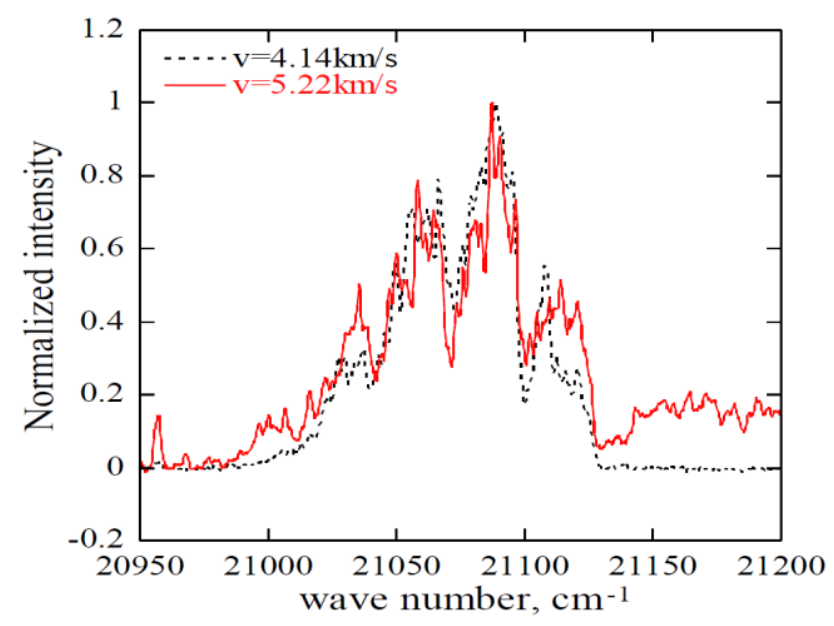

Fig. 8. Experimental spectra by shock wave velocity (Initial pressures: $2666 \mathrm{~Pa}$ and $1333 \mathrm{~Pa}$.) above $21150 \mathrm{~cm}^{-1}$ in these spectra. This part is clearly found at the spectrum of shock wave velocity of $5.22 \mathrm{~km} / \mathrm{s}$, but is not found in the spectral data for shock wave velocity of $4.14 \mathrm{~km} / \mathrm{s}$. Non-resonant part seems to be eliminated by reducing shock wave velocity $1 \mathrm{~km} / \mathrm{s}$. In this way the difference and feature of the measured spectra are figured out, but it is still unclear that the difference is originated from the different vibrational and rotational temperatures. So, they will be studied hereafter.

\subsection{Temperature diagnostic result (Mach number 15)}

The measured spectroscopic data together with theoretical spectrum are shown in Fig.9. The measured data are the same as the spectrum of shock velocity $5.22 \mathrm{~km} / \mathrm{s}$ in Fig. 8 with $6.47 \mathrm{~mm}$ behind the wave front. The theoretical spectrum was calculated with varying four parameters, $T_{v}, T_{r}, \Gamma$ and $\chi_{n r}$ After the fitting, the vibrational and rotational temperatures are estimated to be $4500 \mathrm{~K}$ and $4500 \mathrm{~K}$, respectively. The measured spectrum successfully corresponds to the theoretical spectrum between $21030 \mathrm{~cm}^{-1}$ and $21100 \mathrm{~cm}^{-1}$. Some mismatch can be seen at lower $21030 \mathrm{~cm}^{-1}$ and higher $21100 \mathrm{~cm}^{-1}$. Then, there exists large discrepancy at non-resonant part more than $21130 \mathrm{~cm}^{-1}$. If theoretical spectrum is recalculated to match the difference, new mismatch is generated in other parts. So, the parameters in the fittings program should be configured by more precisely setting.

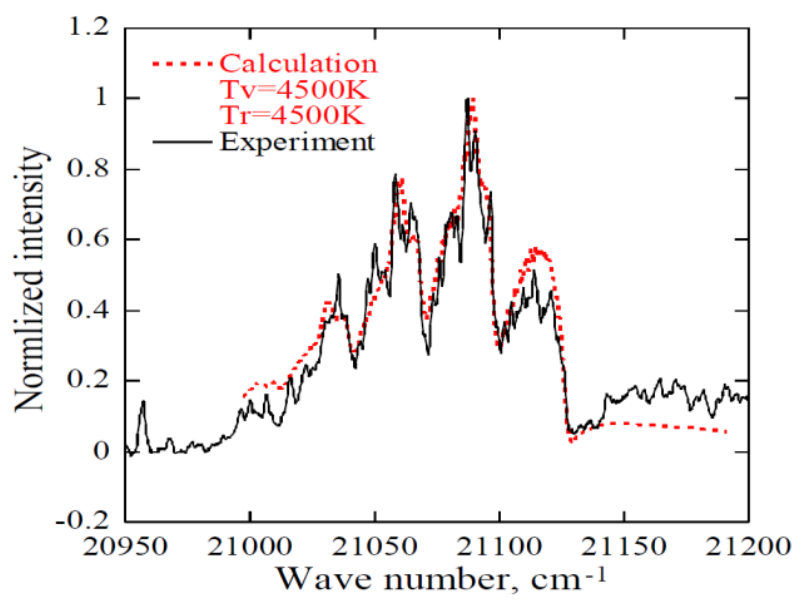

Fig. 9. Fitting of calculated spectrum to experimental spectrum (Initial pressure:1333 Pa)

\subsection{Temperature diagnostic result (Mach number 12)}

The measured spectral shape with theoretical spectrum is shown in Fig.10. This is same as the spectrum Experiment1 of lower velocity in Fig.7 with $5.1 \mathrm{~mm}$ behind the wave front. The theoretical spectrum has been calculated with varying four parameters, $T_{v}, T_{r}, \Gamma$ and $\chi_{n r}$. After the fitting, the vibrational and rotational temperatures are estimated to be $5000 \mathrm{~K}$ and $5500 \mathrm{~K}$, respectively. In the same way as the result of shock wave velocity $5.22 \mathrm{~km} / \mathrm{s}$, the measured spectra can successfully correspond to the theoretical spectrum between $21050 \mathrm{~cm}^{-1}$ and $21100 \mathrm{~cm}^{-1}$. Some discrepancy, however, is found around the peaks of $21030 \mathrm{~cm}^{-1}$ and $21120 \mathrm{~cm}^{-1}$. The spectra are absolutely corresponding over $21150 \mathrm{~cm}^{-1}$ of spectrum, even without non-resonant part. Agreement in 
higher wave number range means more accurate temperature results in our experiment. It is expected that vibrational and rotational temperatures at no non-resonant part are higher than existing non-resonant part. This is because that the measurement locations are different.

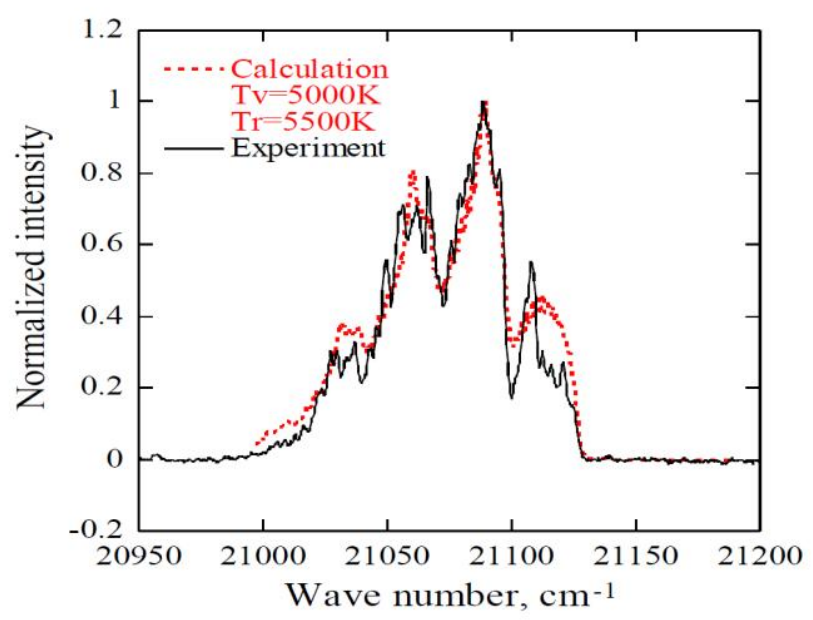

Fig. 10. Fitting of calculated spectrum to experimental spectrum (Initial pressure: $2666 \mathrm{~Pa}$ )

\section{Summary}

The CARS signals have been successfully observed at shock wave velocity range from $4 \mathrm{~km} / \mathrm{s}$ and over $5 \mathrm{~km} / \mathrm{s}$. The measured spectra agree well with the theoretically fitting spectrum. Vibrational and rotational temperatures are decided for nitrogen molecules in air behind the strong shock wave front by the spectral fitting method. Reproducible data can be obtained at similar experimental conditions. The spectrum that does not indicate the non-resonant part has been obtained at initial pressure of 2666Pa for Mach number around 12. The nitrogen molecules in strongly shock-heated air come to these non-equilibrium states between shock front and measured positions with vibrational/rotational temperatures of much lower values than the translational temperature. In our experimental results the nitrogen molecules would come to near ro/vibrational equilibrium situation behind the shock front, because the vibrational temperature almost corresponds to the rotational temperature in shock wave velocity $5.22 \mathrm{~km} / \mathrm{s}$, with $6.47 \mathrm{~mm}$ behind shock front, and shock wave velocity $4.14 \mathrm{~km} / \mathrm{s}, 5.1 \mathrm{~mm}$ behind shock front. It is necessary to compare each temperature at non-equilibrium state with different stronger shock wave velocities with varying the measuring positions behind the strong shock wave front.

\section{References}

1) Honma, $\mathrm{H}$ and lizuka, H.: Experimental and Numerical Studies of Radiation Emission from High Temperature Air behind $10 \mathrm{~km} / \mathrm{s}$ Shock Waves, SAE Technical Paper Series 912025, Re-printed from International Pacific \& Space Technology Conference and 29th Aircraft Symposium Proceedings, Gifu, Japan, 1991.
2) Sharma, S. P. and Gillespie, W. D.: Non-equilibrium and Equilibrium Shock Front Radiation Measurements, J. of Thermophysics and Heat Transfer, 5 (1991), pp. 257-265.

3) Koreeda, J., Ohama, Y. and Honma, H.: Time Resolved Imaging Spectroscopy of Nonequilibrium Shock-front Radiation in Air, Proc. of the $20^{\text {th }}$ International Symposium on Shock Waves, CA, USA, 1995, pp. 1181-1186.

4) Morioka, T., Sakurai, N., Maeno, K. and Honma, H.: Imaging Spectroscopy of Radiation behind Strong Shock Waves in Air, Theoretical and Applied Mechanics, 46 (1997), pp. 241-248.

5) Rado, W. G.: Nonlinear Third Order Dielectric Susceptibility Coefficients of Gases and Optical Third Harmonic Generation., Applied Physics Letters, 11 (1967), pp. 123-125.

6) Eckbreth, Alan C.: BOXCARS: Crossed-beam Phase-matched CARS Generation in Gases, Applied Physics Letters, 32 (1978), pp. 421-423.

7) Eckbreth, A. C., Dobbs, G. M., Stufflebeam, J. H. and Tellex, P. A.: CARS Temperature and Species Measurements in Augmented Jet Engine Exhausts, Applied Optics, 23 (1984), pp. 1328-1339.

8) Magre, P., Collin, G., Pin, O., Badie, J. M., Olalde, G. and Cl'ement, R: Temperature Measurements by CARS and Intrusive Probe in an Air-hydrogen Supersonic Combustion, International Journal of Heat and Mass Transfer, 44 (2001), pp. 4095-4105.

9) Hall, R. J.: CARS Spectra of Combustion Gases, Combustion and Flame, 35 (1979), pp. 47-60.

10) Toyoda, K., Sato, H., Ando, H., Miyazaki, K., Takada, K. and Maeno, K. : Preliminary Test of Coherent Anti-Stokes Raman Spectroscopy for Strong Shock Waves, AIAA Aerospace Meeting, Reno, AIAA Paper No. 2005-0832, 2005.

11) Honma, H., Tsukahara, M., Morioka, T., Katano, T. and Watanabe, N.: Characteristics of a Free-piston Double-diaphragm Shock Tube, $J$ of the Japan Society for Aeronautical and Space Science, 45 (1997), pp. 533-539 (in Japanese). 